\title{
Chronischer Koronarverschluss
}

\section{Bei linksventrikulärer Dysfunktion lohnt eine PCI}

Bei Patienten mit eingeschränkter linksventrikulärer Funktion scheint eine katheterbasierte Wiedereröffnung chronischer Koronarverschlüsse (chronic total occlusion, CTO) die Herzfunktion verbessern zu können. Das könnte sich auf längere Sicht auch prognostisch als vorteilhaft erweisen.

Als CTO bezeichnete komplette Verschlüsse von Koronararterien stellen die auf Revaskularisation bedachten Herzkatheter-Spezialisten vor große Herausforderungen. Der klinische Nutzen der häufig sehr komplexen perkutanen Koronarinterventionen zur CTO-Rekanalisation (CTO-PCI) ist nach wie vor umstritten.

Eine neue Studie rückt nun eine Patientengruppe stärker in den Blickpunkt, die von solchen Herzkatheter-Eingriffen symptomatisch, funktionell und möglicherweise auch prognostisch profitieren könnte, nämlich Patienten mit CTO und ischämisch bedingter Störung der linksventrikulären Funktion. Bei ihnen hatte eine CTO-PCI einen deutlichen Anstieg der zuvor erniedrigten linksventrikulären Ejektionsfraktion (LVEF) zur Folge. Da die LVEF bekanntlich ein starker Prädiktor für das Sterberisiko ist, könnte diese funktionelle Verbesserung auch zu einer Mortalitätssenkung führen.

\section{Hohe prozedurale Erfolgsrate}

Im Rahmen der aktuellen prospektiven Multicenter-Studie, an der auch Kardiologen des Universitäts-Herzzentrums Freiburg-Bad-Krozingen maßgeblich mitgewirkt haben, sind 839 Patienten konsekutiv einer elektiven CTO-PCI unterzogen worden. Je nach LVEF sind diese Patienten in drei Gruppen unterteilt worden: LVEF $>50 \%$ (Gruppe 1), LVEF 35-50\% (Gruppe 2), LVEF $<35 \%$ (Gruppe 3).

Die relativ kleine Gruppe 3 mit deutlich eingeschränkter linkventrikulärer systolischer Funktion umfasste 72 Patienten $(8,6 \%)$. Im Vergleich zur Gruppe mit normaler LVEF (Gruppe 1) hatten diese insgesamt kränkeren Patienten mehr Begleiterkrankungen wie Diabetes und PAVK, zudem bestand bei ihnen häufiger eine koronare 3-Gefäßerkrankung. Auch klagten sie in stärkerem Maß über eine schwere Dyspnoe und seltener über Angina pectoris als Patienten mit normaler oder nur gering eingeschränkter linksventrikulärer Funktion.

Die Rate für den anhand angiografischer Kriterien beurteilten Erfolg der CTO-PCIProzeduren, die an drei europäischen Zentren von erfahrenen CTO-Experten durchgeführt wurden, war mit 93,6\% hoch und in allen drei Gruppen nahezu gleich. Die Dauer der klinischen Nachbeobachtung betrug im Mittel 16 Monate.

\section{Wie häufig kamen kardiovaskuläre Ereignisse vor?}

Die Raten der Patienten, die zwei Jahre nach der CTO-PCI noch am Leben waren, ohne dass bei ihnen ein schwerwiegendes kardiales oder zerebrovaskuläres Ereignis (MACCE: major adverse cardiac or cerebrovascular event) aufgetreten war (MACCE survival rate), waren mit 86\% (Gruppe 1) vs. $82,8 \%$ (Gruppe 2) vs. $75,2 \%$ (Gruppe 3) nicht signifikant unterschiedlich. In allen drei Gruppen war eine erfolgreiche CTO-PCI im Vergleich zu einer erfolglosen Intervention generell mit einer niedrigeren MACCE-Rate assoziiert (16,6\% vs. $50,0 \%$ ). Dementsprechend erwies sich neben den Faktoren Alter und Diabetes mellitus auch ein „Versagen“ der CTO-PCI als unabhängiger Prädiktor für das Auftreten von kardiovaskulären Ereignissen. Die LVEF war dagegen im relativ kurzen Beobachtungszeitraum der Studie ohne prädiktive Bedeutung.

In der Gruppe 3 mit eingeschränkter linksventrikulärer Funktion wurde die LVEF nach sechs Monaten erneut bestimmt. Dabei zeigte sich, dass es bei denjenigen Patienten, die einer erfolgreichen

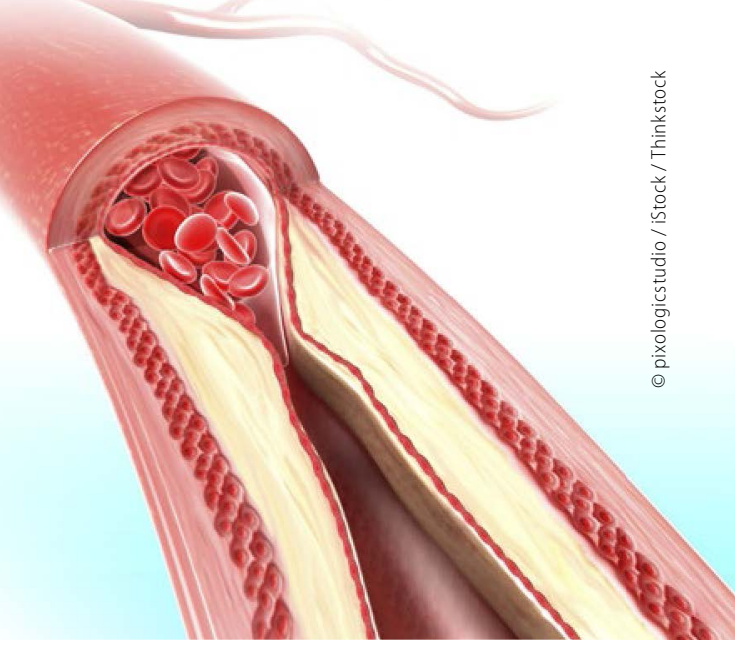

Komplette Verschlüsse von Koronararterien stellen Herzkatheter-Spezialisten vor große Herausforderungen.

CTO-PCI unterzogen werden konnten, zu einem signifikanten Anstieg der LVEF von initial $29,1 \%$ auf $41,1 \%$ gekommen war. Gleichzeitig war bei ihnen eine deutliche Verbesserung der Dyspnoe-Symptomatik $\mathrm{zu}$ verzeichnen.

\section{Weitere Studienergebnisse}

Das an der Studie beteiligte Kardiologenteam des Universitäts-Herzzentrums Freiburg-Bad-Krozingen um Dr. Dr. Kambis Mashayekhi und Prof. Franz-Josef Neumann hat jüngst mit REVASC eine weitere Studie zum Nutzen der CTO-PCI vorgestellt. Im Blickpunkt dieser Studie stand ebenfalls die potenzielle Verbesserung der linksventrikulären Funktion durch CTOWiedereröffnung per PCI. Doch konnte eine entsprechende funktionelle Verbesserung in diesem Fall nicht nachgewiesen werden. Das könnte daran gelegen haben, dass die linksventrikuläre Funktion der Patienten mit CTO-PCI - bei ihnen betrug die LVEF schon zu Beginn im Mittel 55\% - noch relativ gut war.

Peter Overbeck

Galassi A. R. et al. JACC Cardiovascular Interventions 2017, https://doi.org/10.1016/j.jcin.2017.06.058 